Cell Research (2002); 12(3-4):247-255

http://www.cell-research.com

\title{
A Pin gene families encoding components of auxin efflux carriers in Brassica juncea
}

\author{
Wei Min NI ${ }^{1,2}$, Xiao Ya $_{\text {CHEN }}^{1}$, Zhi Hong XU ${ }^{1,2}$, Hong Wei XUE ${ }^{1,2, *}$ \\ 1 National Laboratory of Plant Molecular Genetics, Institute of Plant Physiology and Ecology, Shanghai Institutes \\ for Biological Sciences, Chinese Academy of Sciences \\ ${ }^{2}$ Partner Group of Max-Planck-Institute of Molecular Plant Physiology (MPI-MP) on "Plant Molecular Physiology \\ and Signal Transduction ", 300 Fenglin Road, Shanghai 200032, China
}

\begin{abstract}
Based on the sequence information of Arabidopsis PIN1, two cDNAs encoding PIN homologues from Brassica juncea, Bjpin2 and Bjpin3, were isolated through cDNA library screening. Bjpin2 and Bjpin3 encoded proteins containing 640 and 635 amino acid residues, respectively, which shared $97.5 \%$ identities with each other and were highly homologous to Arabidopsis PIN1, PIN2 and other putative PIN proteins. BjPIN2 and BjPIN3 had similar structures as AtPIN proteins. Northern blot analysis indicated that Bjpin2 was expressed in stem, leaf and floral tissues, while Bjpin3 was expressed predominantly in stem and hypocotyls. Two promoter fragments of pin genes, Bjpin-X and Bjpin-Z, were isolated by 'genome walking' technique using primers at 5' -end of pin cDNA. Promoter-gus fusion studies revealed the GUS activities driven by Bjpin-X were at internal side of xylem and petal; while those driven by Bjpin-Z were detected at leaf vein, epidermal cell and cortex of stem, vascular tissues and anther. Results of the pin genes with different expression patterns in B. juncea suggested the presence of a gene family.
\end{abstract}

Key words: Brassica juncea, polar auxin transport, auxin efflux carrier, promoter.

\section{INTRODUCTION}

Auxin is critical for plant embryo development, morphogenesis and especially polar differentiation and growth. Polar auxin transport (PAT), detected in stem and roots of all higher plants, was involved in plant apical dominance, tropic growth, vascular and floral tissue differentiation, plant pattern for-

* Corresponding author: Dr. Hong Wei XUE, Institute of Plant Physiology and Ecology, Shanghai Institutes for Biological Sciences, Chinese Academy of Sciences, 300 Fenglin Road, Shanghai 200032, China

Tel: +862164042090 ext 4411, Fax: +86 2164042385 ,

E-mail: hwxue@iris.sipp.ac.cn

**The nucleotide sequences reported in this paper have been submitted to the GenBank ${ }^{T M}$ / EMBL Data Bank with accession numbers AJ249297 ( Bjpin2 cDNA), AJ249298 (Bjpin3 cDNA), AJ308229 (Bjpin-X) and AJ308230 (Bjpin-Z).

Received May-13-2002 Revised July-22-2002 Accepted July-252002 mation and lateral root development, etc (for reviews, seeNi, et al, 2000[1]; Berleth and Sachs, 2001[2]). Current model of PAT, chemiosmotic hypothesis (Rubery and Sheldrade[3] and Raven[4]), posited the occurrence of PAT through the action of cellular auxin influx and efflux carriers locating on the plasma membrane of transporting cells, and the direction of PAT depends on the basal location of the efflux carriers.

The first molecular support of the model came from the characterization of a candidate auxin influx carrier, AUX1 (auxin resistant1), which was a presumptive membrane protein similar to amino acid permeases[5] and specifically expressed in the root. Further isolation and characterization of two genes, Atpin1 and Atpin2, indicated that the primary func- 
tion of the AtPIN1 protein was for PAT in the inflorescence axis and AtPIN2 in root tissues[6-10]. AtPIN1 involved in the auxin transport from stem to root tip and was critical for symmetry differentiation of embryo, the initiation and radial position of plant lateral organs, pattern formation of leaf vein [11-13]. AtPIN2 played a role in root gravitropic response together with AUX1[9]. AtPIN1 was a membrane protein locating at the basal end of auxin transport-competent cells in vascular tissues[7]. AtPIN2-expressing yeast cells were resistant to toxic fluoroindoles, an IAA analogy[8], and released preloaded IAA more rapidly than control cells demonstrated that AtPIN2 transported IAA out of the yeast cells and indeed a component of auxin efflux carriers[9]. The Arabidopsis mutant pin 1 showing normal tropic responses of shoot and root[10] also indicated that there maybe other pin genes controlling the lateral auxin transport pathways. Presence of other cDNAs encoding PIN homologues indicated a multi-gene family in Arabidopsis genome that, however, suggested the specific roles of pin genes in auxin related plant growth and development. Analyses of Atpin1 (widely expressed in all the tissues tested) and Atpin2 (specifically expressed in the root) indicated their expression patterns were closely linked to their physiological functions. As a conclusion, isolation of pin genes, especially studies on their expression patterns will provide informations on functional mechanisms of PAT during plant growth and development, especially polar differentiation.

We are interested in the functions of PAT in plant cells. From Brassica juncea we have isolated an auxin efflux carrier (BjPIN1) and performed functional characterization through transgenic approaches of Arabidopis[14]. Here we report the isolation of two putative pin-gene promoters and two cDNAs encoding PIN homologous of B. juncea, as well as the studies on their expression pattern analysis.

\section{MATERIALS AND METHODS}

\section{Materials}

Enzymes used for DNA restriction and modification were obtained from Boehringer (Mannheim, Germany). DNA primers for polymerase chain reaction (PCR) and "LAPCR in vitro Cloning” kit used for 'genome walking' were obtained from
TaKaRa Biotechnology (Dalian, China). 5-bromo-4-chloro-3indolyl b-D-glucuronic acid (X-Gluc) was obtained from Sigma (USA). Radiochemicals [ $\left.\alpha{ }^{32} \mathrm{P}\right] \mathrm{dCTP}$ was obtained from Yahui Company (Beijing, China). The leaf IZAPII cDNA library of Brassica juncea was kindly provided by Pua E-C. (National University of Singapore).

\section{Bacteria and plants}

Escherichia coli strain XL-1 Blue (Stratagene, La Jolla, USA) was used for library screening and DNA cloning. Agrobacterium strain LBA4404 was used for plant transformation. B. juncea (Indian mustard) and Nicotiana tabacum plants were grown in the green house with a $12 \mathrm{~h}$ light $\left(26^{\circ} \mathrm{C}\right)$ and $12 \mathrm{~h}$ dark $\left(20^{\circ} \mathrm{C}\right)$ period.

\section{Isolation of the Bjpin cDNAs}

Bjpin cDNAs were isolated as described[14]. In short, two reverse degenerated primers were designed based on the nucleotide sequences of Atpin1[8] and two other putative pin sequences and used to amplify the Pin-homologous cDNA fragments from cDNA library of B. juncea. Specific primers were then used to isolate the full-length cDNAs through PCR-based screening[14], [15].

DNA sequencing was performed by TaKaRa Biotechnology (Dalian, China). Computational analysis was performed with the help of the programs of the Wisconsin Genetics Computer Group (GCG Package, Version 10.1). The BLAST search programs were used for sequence comparisons on DNA and amino acid sequences in GeneBank, EMBL, dbEST and SwissProt databases.

\section{Northern blot analysis}

RNAs were prepared from well-watered B. juncea plants according to the protocol of Logemann et al[16] and quantitiated spectrophotometrically at $260 \mathrm{~nm}$. RNA (20 $\mu$ g per lane) was electrophoretically separated in denaturing agarose gels and blotted onto Hybond- $\mathrm{N}^{+}$membranes (Amersham, USA). RNA was fixed onto the membrane by heating at $80^{\circ} \mathrm{C}$ for $2 \mathrm{~h}$. PCR products of around 400-bps of the Bjpin2 and Bjpin3 (encoded the amino acid residues at positions 305-441 and 305-436, respectively) were served as $\left[\alpha_{-}{ }^{32} \mathrm{P}\right] \mathrm{dCTP}$-labeled hybridization probe. Membranes were hybridized and washed as described[17]. Blots were exposed to Fuji X-films between intensifying screens for 3-4 d at $-70^{\circ} \mathrm{C}$.

\section{Isolation of the promoter regions of pin genes}

Promoter regions of pin genes were isolated using "LAPCA in vitro cloning" kit. $5 \mathrm{mg}$ genomic DNA was digested with Hind III or EcoR I and then ligated with corresponding adaptors. Ligation products was then denatured at $94^{\circ} \mathrm{C}$ for $5 \mathrm{~min}$ and used as templates for 1 st round PCR with primers C1 (5'GTACATATTGTCGTTAGAACGCGTAATACGACTCA-3') and BjPS1 (5'-TACAGAGCCGTAGGCGAGGATCATAG-3'). PCR was performed as follows: $94 \mathrm{oC}$ for $30 \mathrm{sec}$; $58 \mathrm{oC}$ for $90 \mathrm{sec} ; 72^{\circ} \mathrm{C}$ for $3 \mathrm{~min}$; 30 cycles. PCR products were then diluted for 10 times and $1 \mathrm{ml}$ was used as templates for 2nd PCR using primers C2 (5'CGTAATACGACTCACTATAGGGAGA-3') and BjPS2 (5'- 
CGAGGATCATAGCGACGTAGAG-3') under same conditions as described above. PCR products were separated on the agarose gel and subcloned into TA vectors. Colonies harbouring PCR products were sequenced (TaKaRa Biotechnology, Dalian) and analyzed with programs of the Wisconsin Genetics Computer Group (GCG Package, Version 10.1) and PLACE.

Plant transformation with binary constructs harbouring promoter regions of pin genes

Promoter regions before ATG of Bjpin-X was amplified via PCR using standard primer SP6 and GW1.2A (5' CTTATTTACGGTGGAATATAGAGGG-3' ) with Pfu taq polymerase and then digested with Hind III, resulted DNA fragment was then ligated to pBSK precut with Sma I/Hind III. Resulted plasmid DNA was digested with Hind III / BamH I and DNA fragment was ligated to pBI101.3 cut with same enzymes.

Same cloning strategy was used for Bjpin-Z, primers SP6 and Bjpin1S (5'-CTTCTTTCTGGTTGATTAGAGAG-3') were used for amplification, restriction enzyme Sal I was used for cloning procedures.

The resulted plasmids, containing the promoter-uidA coding region, were transformed into A. tumefaciens strain LBA4404 for tobacco transformation. Transformation, using leaf disc as materials for transformation, were performed as described.

\section{Analysis of transgenic plants}

Transgenic tobacco plants were confirmed via PCR using primers located in promoter region, for Bjpin-X, primers GW1.2S (5' -CTTCTGCTCTTGCATGATCTTCG-3' ) and W1.2A (see above), for Bjpin-Z, primers Bjpin1S (see above) and GW07A (5' TATTATCGCCGGATGAGTGGCA-3' ) were used. PCR was performed as follows: $94^{\circ} \mathrm{C}$ for $5 \mathrm{~min}, 1$ cycle; $94^{\circ} \mathrm{C}$ for $1 \mathrm{~min}, 55^{\circ} \mathrm{C}$ for $1 \mathrm{~min}, 72^{\circ} \mathrm{C}$ for $1 \mathrm{~min}, 35$ cycles; with a final extension at $72^{\circ} \mathrm{C}$ for $10 \mathrm{~min}$.

GUS activities were determined using 5-bromo-4-chloro-3indolyl b-D-glucuronic acid (X-Gluc) as substrate[18].

\section{RESULTS}

Isolation of Bjpin2 and Bjpin3 cDNAs from $B$. juncea

Using degenerated primers, a DNA fragment with a size of $550 \mathrm{bp}$ was amplified and sequenced, the deduced peptide sequence showed $75 \%$ identi- ties with isolated AtPIN1 at position $1 \sim 145$, indicating a putative PIN-like fragment of B. juncea. PCR-based screening of B. juncea cDNA library was then performed and two cDNAs, Bjpin2 and Bjpin3, were isolated. There were in-frame stop codens before the translation start code 'ATG' in both cDNAs, which indicated that full-length open reading frames were included. The predicted proteins of BjPIN2 and BjPIN3 contained 640 and 635 amino acid residues respectively, and had a molecular mass of $70 \mathrm{kDa}$, similar to that of previously reported AtPIN1[6] and AtPIN2/EIR1 [8],[9]. The calculated isoelectric points of BjPIN2 and 3 were around 8.8.

BjPIN2 and BjPIN3 shared 97.5\% homologies with each other and were highly homologous to Arabidopsis PIN proteins (Tab 1), with the highest identities (93\%) with AtPIN3 (accession No. AF087818).

\section{Structural organization of the BjPIN proteins}

Fig 1 shows the alignment of BjPIN2 and BjPIN3 with BjPIN1, AtPIN1, AtPIN2 and other PIN-like proteins in Arabidopsis. Comparison of all the proteins revealed the presence of conserved regions locating at both $\mathrm{N}$ - and C-terminus, which are highly hydrophobic. Presence of around 11 predicted transmembrane regions in BjPIN2 and BjPIN3 was similar with that in AtPIN1. A non-conserved central region is predominantly hydrophilic.

\section{Expression pattern analyses of Bjpin2 and Bjpin3}

Northern blot analysis was performed to detect the transcription levels of both Bjpin2 and Bjpin3. The DNA fragments encoding the non-conserved (varied) regions of Bjpin2 and Bjpin3 were served as hybridization probes. As shown in Fig 3A and Fig $3 \mathrm{~B}$, single band of transcripts of approximately $2.5 \mathrm{~kb}$, consistent with the length of both $\mathrm{cDNAs}$, was detected. Bjpin2 mRNA was detected in stem, leaf, and floral tissues, and the Bjpin3 mRNA predominantly in stem and hypocotyls. No detectable mRNA was found in root tissues.

Tab 1. Amino acid sequence comparison of BjPIN2 and BjPIN3 to isolated AtPIN1, 2 and other PIN-like proteins of Arabidopsis. Numbers indicated percentages of identical and similar amino acids. Accession numbers for AtPIN1, 2, 3, 4, 6 and 7 refer to Fig 1. I: identities; S: similarities.

\begin{tabular}{ccccccc}
\hline I/S & AtPIN 1 & AtPIN2 & AtPIN3 & AtPIN4 & AtPIN6 & AtPIN7 \\
\hline BiPIN2 & $64 / 81$ & $62 / 77$ & $93 / 97$ & $82 / 85$ & $53 / 72$ & $87 / 92$ \\
BiPIN2 & $64 / 82$ & $62 / 76$ & $93 / 97$ & $82 / 85$ & $53 / 72$ & $87 / 92$ \\
\hline
\end{tabular}




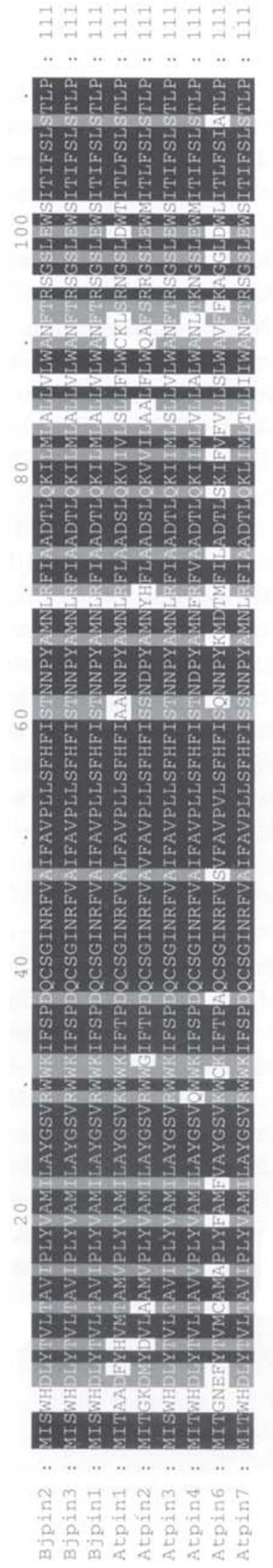

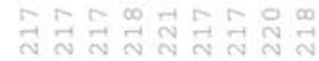

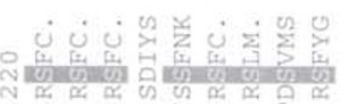
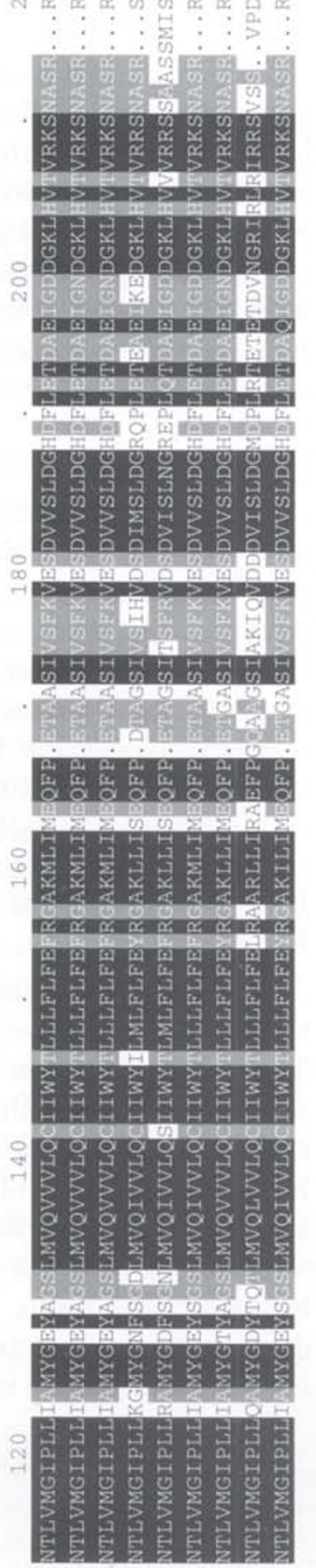

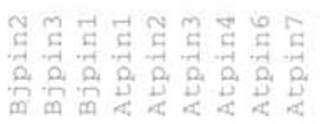
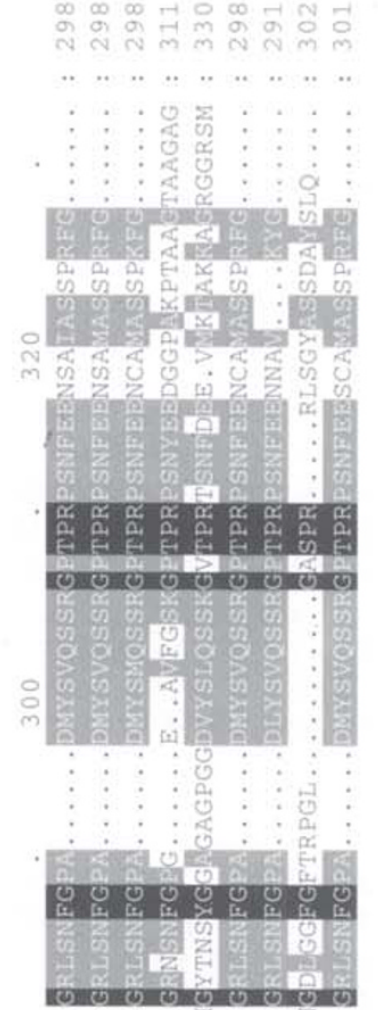

$$
\infty
$$
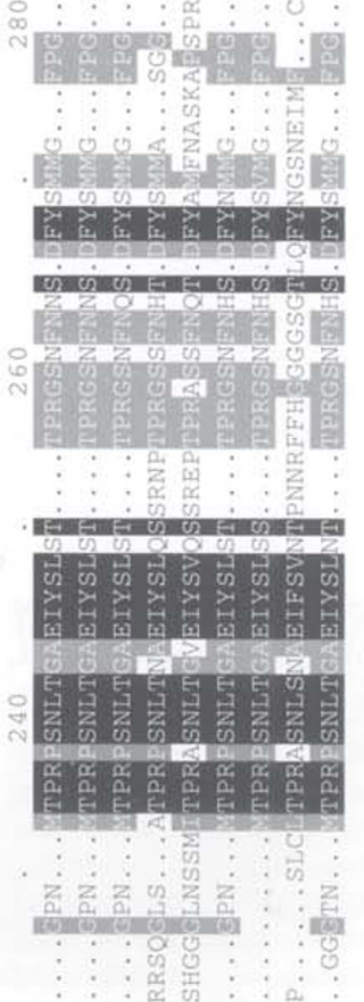

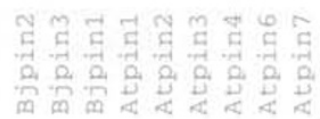
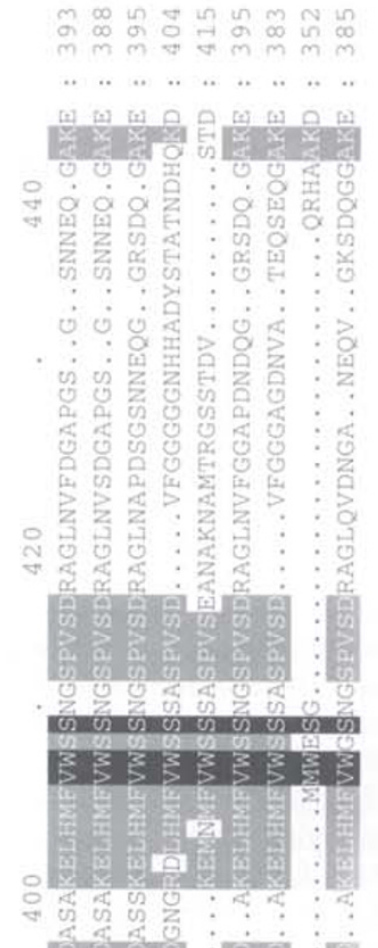

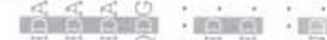
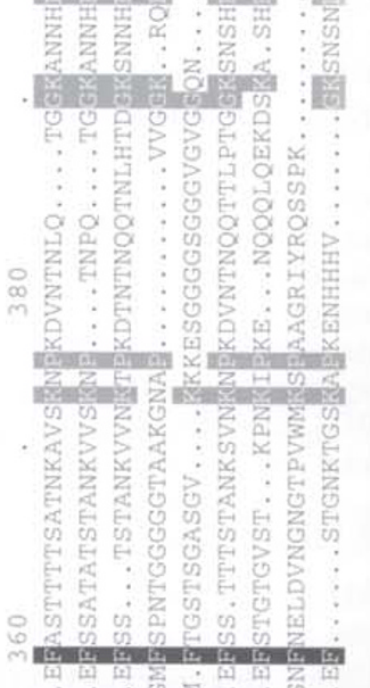

ㄴ.

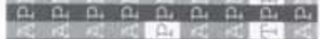

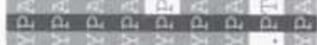

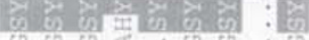

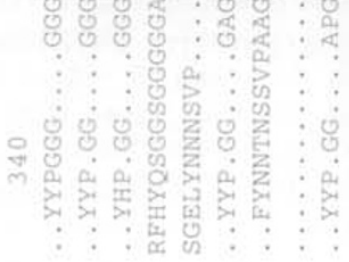

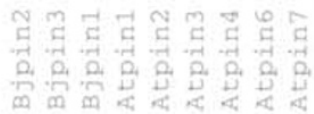


Wei Min NI et al
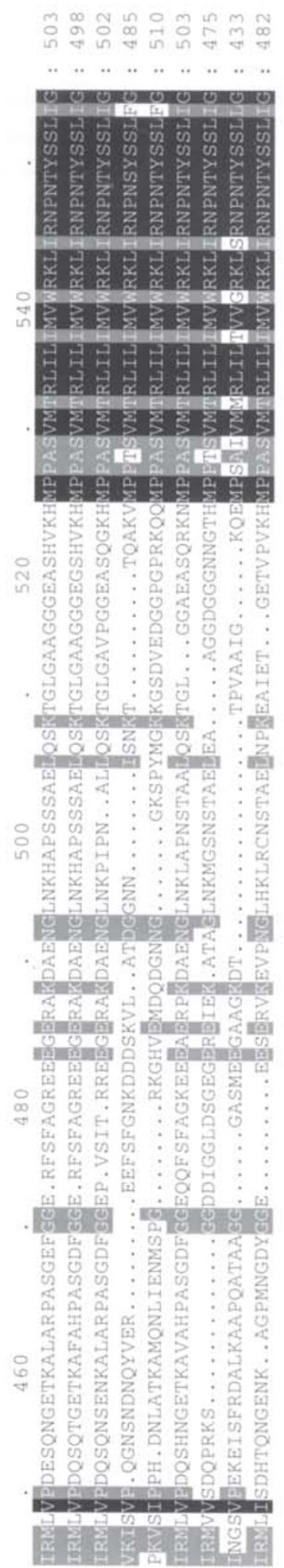

$\begin{array}{lllllllll}. . & . & . . & . . & . . & . . & . & . . & . .\end{array}$
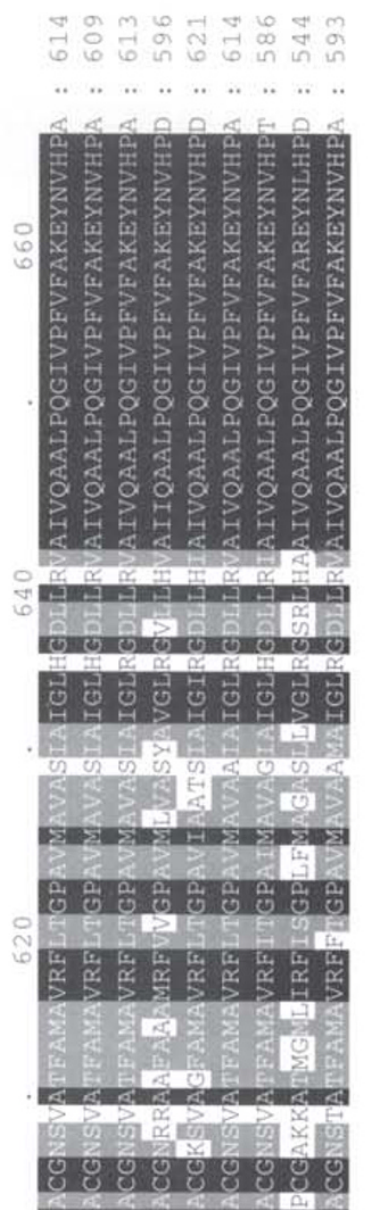

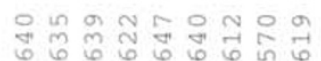
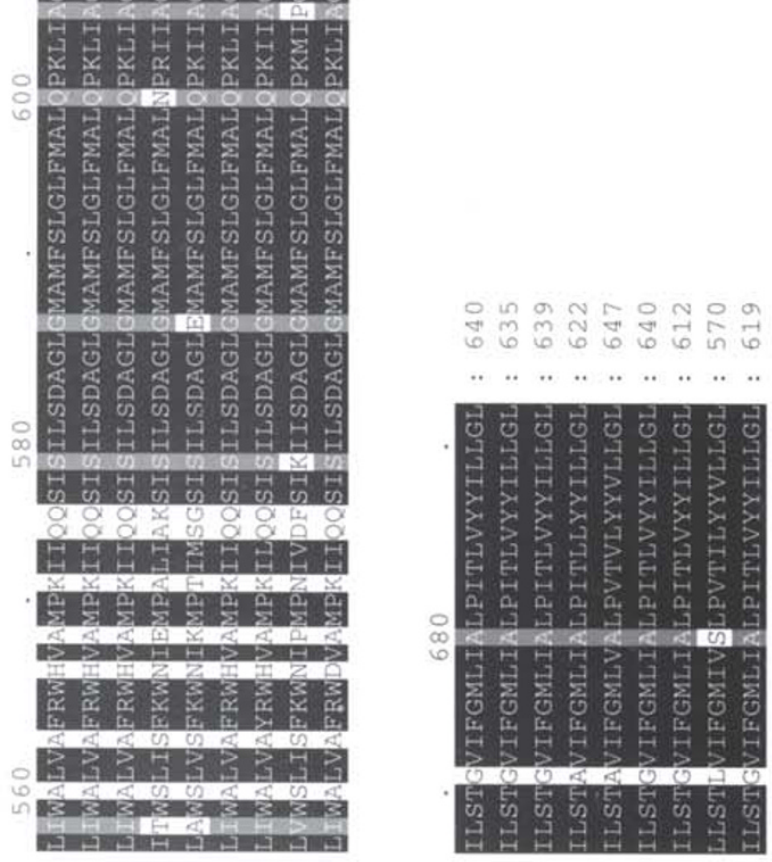

究完

宬

कै

峁

艺

$\begin{array}{cc}0 & \infty \\ 0 & 0 \\ 0 & \infty \\ 0 & \infty\end{array}$

थ

령 द्वे

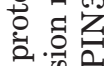

云造走

它

究部

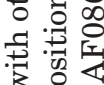

s.

总胥

象虽

चี สี

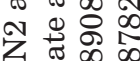

党.

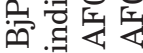

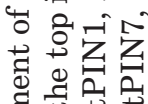

严焉艺

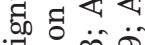

艺 की

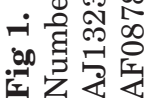


Isolation of promoter regions of Bjpin-X and Bjpin$Z$, which encoded other two isoforms of pin family from B. juncea

Two promoter fragments, Bjpin-X and Bjpin-Z, were isolated through genome walking technique based on the cDNA sequence of Bjpin1. After amplification using primers within adaptor and specific primers designed at 5' -coding region of Bjpin1, two DNA fragments, $1.2 \mathrm{~kb}$ (Bjpin-X, Fig 2A) and 0.7 kb (Bjpin-Z, Fig 2B), respectively, were amplified and sequenced. Comparison analyses showed that the coded peptides by Bjpin-X and Bjpin-Z, as well as their 5'- untranslated regions shared high homology with isolated Bjpin1, Bjpin2 and Bjpin3 but still with differences, which indicated that Bjpin-X and Bjpin-Z were promoter regions of other 2 pin genes from B. juncea than Bjpin1, Bjpin2 and Bjpin3. Sequence analyses further revealed the presence of basic elements "TAAT" and "CAAT", and of particular interests, two pollen specific elements “AGAAA” in Bjpin-Z (Fig 2B).

\section{Expression pattern analyses of Bjpin-X and Bjpin-Z}

Both promoter regions of Bjpin-X and Bjpin-Z were subcloned into binary vector pBI101.3, and the resulted Bjpin-X::GUS and Bjpin-Z::GUS constructs were used for agrobacterium-mediated tobacco transformation. Comfirmation of resistant plants via PCR amplification indicated that both Bjpin-X::GUS and Bjpin-Z::GUS had been integrated into tobacco genome (Fig 3C). At least 10 independent transgenic tobacco plants were used for GUS activity analyses. GUS activities, driven by Bjpin-X, were detected at xylem region (Fig 3D, E) and petal (Fig 3F); while those driven by Bjpin-Z were detected in mature anther and pollen (Fig 3G), leaf vein (Fig $3 \mathrm{H}, \mathrm{I}$ ), epidermis, cortex of stem (Fig 3J), and vascular tissues (Fig 3J, K).

\section{DISCUSSION}

Till now, at least 8 different pin-like genes have been detected in Arabidopsis genome and a few homologous genes in rice as well. Presence of multiisoform auxin carriers, based on the extensive functions of auxin, suggest that some pin genes might share functional redundancy, yet each member of the pin gene family has its distinct functions. On the basis of distinct expression pattern and functions of AtPIN1 and AtPIN2/EIR1/AGR1 in the shoot and root, respectively[6-9], it is proposed that the tissueor cell-specific auxin efflux carriers are responsible for multiple auxin transport pathways[19]. Presence of at least 5 pin-like genes in B. juncea, as demonstrated by our studies, indicated a pin-gene family in B. juncea. Different expression patterns of the pin-like genes in $B$. juncea suggest their different roles during developmental processes. Isolation and expression pattern analysis of pin genes will certainly provide hints on their roles during plant growth and development.

Bjpin2 and Bjpin3 were relatively high expressed in stem, which, however, suggests that both proteins might participate in the polar auxin transport in stem and play a specific role in stem or vascular tissue development. Recent studies have shown that AtPIN3 was expressed predominantly in endodermal cells with a lateral localization[20]. Since the isolated BjPIN proteins shows highest identity with AtPIN3, it is reasonable to assume that Bjpin2 and Bjpin3 might have a similar expression pattern with AtPIN3 in stem, and may play a role in growth that require lateral auxin distribution, such as shade avoidance[21]. Different expression patterns of Bjpin2 and Bjpin 3 to isolated Bjpin1[14] and Arabidopsis Atpin1 and Atpin2, together with the difference between themselves, i.e. presence of Bjpin2 in leaf, floral tissues and stem while Bipin3 was relative specific in stem and hypocotyls, also indicated that they might have their distinct functions.

Different expression patterns of Bjpin-X, Bjpin$\mathrm{Z}$ and isolated Bjpin1[14] at xylem (Fig 3E, K, L) indicated their specific roles during vascular development. Further, plant tropism growth was due to the accumulation of auxin at one side epidermis and cortex of stem (response to the stimulations) resulting in the affection of cell growth. Expression of Bjpin-X in both epidermis and cortex of stem, Bjpin-Z only at partial epidermis and cortex of stem, and isolated Bjpin 1 at epidermis and cortex around stem indicated that Bjpin-Z might be involved in the unsymmetrical growth of stem by environmental stimulation such as light and gravity. 
$1 \mathrm{~A}$

CGTAATACGACTCACTATAGGGAGAAGCTTATTCCATGGTGCAGCCTTACACA TGTTTTGAAAATTTGTGCCTCCATCACATAACACCTTTAGATCACTCATCTCT TCGTATGGCAGCTTGTACCAAATTCGTTGTTTGTACATATCCACTATTTCAGC CGAAATAAGGTTACTAAACAGACTGGGAGCCGCAGATTGCAAATAATGGCTTT TCCCTCCTACATAGCAAGTTGTTCCTTCTTTTAGCTCAAACCTCCCCTCATAG TTTACAATGAAAGTGACGTCGATATCACCACTGAAACATTACAAAACAACATC AGTTTGACACAGGAACACAAAAAAAAACTTCATTGACACATTTAATCGAACAC CTTATGTGCATTATATTTTTTCACAACTTTTTCCAATTAATCTCAATTATTC TTGTCCCCAACACTCTAATAGCGTCACTTTAACATTCTTAACAAGAATTCCCA ACTCATAAACCGCGAACCCTTATTTATATGAGTTTCATACCCATTTCTCTGTT GGTGATTTAGAAAGAGAGGTACCTCATCTCGTAGATCTCAAATCGATGTCACT CTCCTCTGATTATATCTTTTGCTTTCAATTTCTTCTAATTATATCTTCTGATT ACTTCCCAAAGTTTCTTCTGCTCTTGCATGATCTTCGTCCCCAAACAACTTAG AGAATCTTCGAGTGTGGAAAGGTAAAGAGAAACAATTTTTTTTTAACTTTGTT GAGAAACGACGTCGTCTAGTTTAAGGGTTTATTATATTTTTTTAAACAAATAA AGTGACAAAATATTTAAGAAAAGCCACATAATCAATTCAAAATGAACAGCTCA GCTTCCGTCACGGCTGAATAACGGAAAATGGGTTTATGTATGATAAGACTATT GTTTGTGTGTTGGTGTATGAAATTTAAATAAATGATGTGTTGGTGTATGTTTT CGCACAGCTAACGTGTTGATGTACTGATCGGGACCGCAGTCCTCTGTTGATGT ATGAATTAGCCGATTTCCCTCTTCTTCATACCCCAACACCGTTTACAAACTTT ACCCTCTATATTCCACCGTAAATAAGATG

1B

GAATTATTATTTTATTATTGTCTAGGGTACTTATATAAATACTGAAATTCGT CATTTAATCATTATACGAAAATGgATTAgTTAgAgAATGTAAA AGAAA GgTG ATGTGAAACTTATACCAAAGCAAAGAGGAGGGGACAGAAG AGAAA AAGGTAT GTCTGCGATGCCACATGTAGACCTTCTTTTAAAGATCTAACTCTATTTTTGCA CATGTAAAACTATTGCAGACTACTTCTGTTTTACATAAGTGTTCCCCAGATAT ATTTTCATCGGCAAGTTTATTTGTATGCTAATTTATTCTTGTATTATAAACTA TACCCTAAAATATTCCATTTATTTACAAATTAATTTTTTGGTCAAATTAGCTA TATAGTCATTTAACCAAACAAATGAAACACTATAGTTACTTACCAAATGACCC AATACACGCGGTATGGTTATTCTACGAGAAGGACAATGACCCTGTTAAATACG ATACTCAATTTTCAAAAGATAAGAAATAAATAATGAATGATAAAAAAGA AACTTTGGGTTGTCAAGTATTTGCTGTATAAAAAGAGGTGTAAGGTGGCTATC ACTCCTTAAATTATATCCTCAGAGCCAAGGTTGCTCACTCCTTCCACACATGC TCCAAGTCCCCGCCTTCTTCTTCTTCTTCAGTCTCCTTTCATACCTCCACACC ATTGCTTAACGTTTTCTCTCTAATCAACCAGAAAGAAGATG

Fig 2. Promoter regions of Bjpin- $X$ and Bjpin- $Z$

A: Bjpin-X; B: Bjpin-Z. ATG: translation start site; CAAT and TAAT: basic elements of promoter region; AGAAA: element for pollen-specific expression. 
A

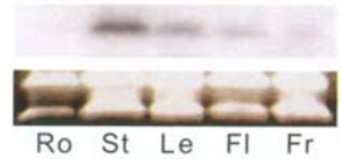

B
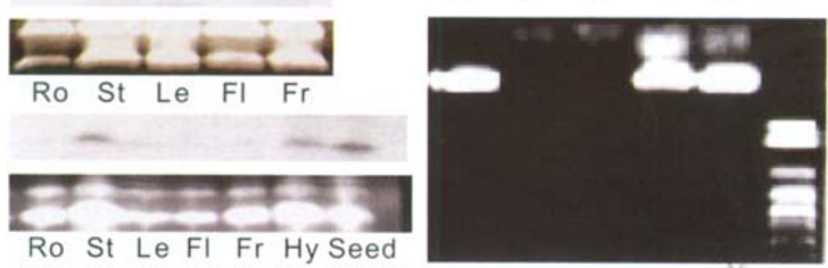

C
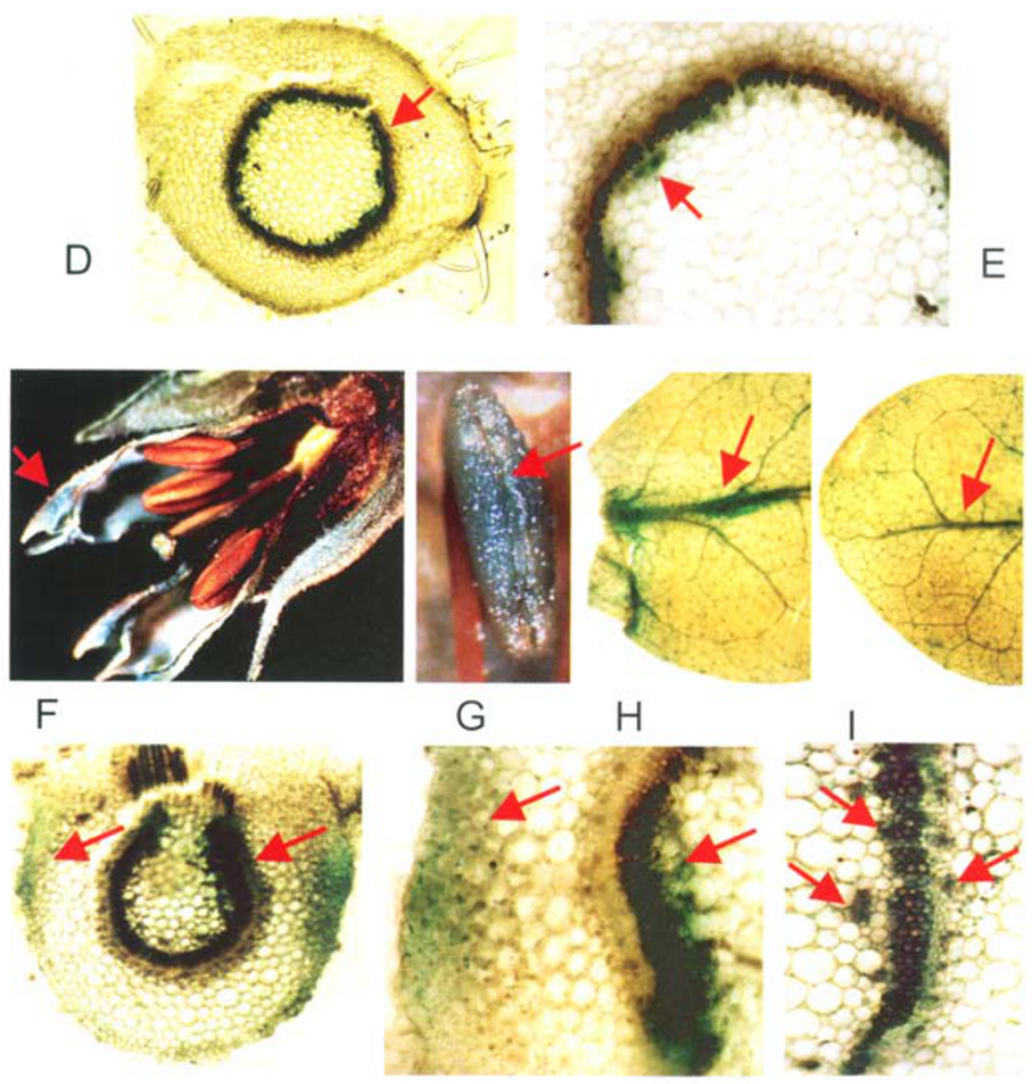

$J$

$\mathrm{K}$

$\mathrm{L}$

Fig 3. Expression pattern analyses of Bjpin genes

Northern blot analysis of Bjpin2 and Bjpin3 transcription levels. A: Bjpin2; B: Bjpin3. Plant tissues were as follows: Ro-root; St-stem; Le-leaf; Fl-flower; Fr-fruit; Hy-hypocotyl; seed. Equal amounts of RNA (shown in bottom lines) were used for hybridization. C: Confirmation of transgenic tobacco plants harbouring Bjpin-Z::GUS construct via PCR. Transformed vector (Lane 1), genomic DNA of untransformed (Lane 2) and transformed plants (Lane 3-5) were used as templates for PCR. M: DNA ladder.

GUS expression detection in transgenic tobacco plants. GUS activities driven by Bjpin-X were detected in stem (D), vascular tissues (E) and petal (F); those driven by Bjpin-Z were detected in anther $(G)$, leaf vein (H, I), epidermis and cortex of stem $(J)$, stem vascular tissues $(\mathrm{K})$; and driven by Bjpin1 were in stem vascular tissues (L). Arrows showed the positions of GUS expressions.

Expression of Bjpin-Z in anther and mature pollen indicated its role in reproductive growth. The relatively high level of auxin in plant anther is critical for anther development, while source of auxin, $i$. e. the biosynthesis site and transport in anther is still unclear. Plant floral tissues originate from leaf and auxin can be synthesized at edge of young leaf then transported to stem through vascular tissues [22],[23]. Recent studies showed that stigma may synthesize auxin and the auxin can be transported to floral base through polar transport resulting in the auxin gradient from stigma to receptacle and 
controlling of morphogenesis of pistil[24]. Expression of Bjpin-Z in anther may suggest the auxin synthesis capacity of pollen, even the role of Bjpin-Z during pollen germination and fertilization processes.

The differences of the expression patterns of Bjpin-Z and Bjpin1 in leaves, i.e. Bjpin-Z was restricted at vascular tissues while Bjpin 1 was mostly at edge of leaves, suggested their different roles in leaf vascular development. Further expression of Bjpin1 at leaf trace indicated its role in auxin transport from leaf to stem.

\section{ACKNOWLEDGMENTS}

Studies were supported by the National Natural Sciences Foundation of China (No. 30070073, 95Yu-29-7) and State Key Project of Basic Research (No. G1999011604). We greatly thank Dr. Klaus Palme for providing the Atpin1 nucleotide sequences.

\section{REFERENCES}

[1] Ni WM, Chen XY, Xu ZH, Xue HW. Advance in study of polar auxin transport. Acta Botanica Sinica 2000; 42:221-8.

[2] Berleth T, Sachs T. Plant morphogenesis: long-distance coordination and local patterning. Curr Opin in Plant Bio 2001; 4:457-62.

[3] Rubery PH, Sheldrake AR. Carrier-mediated auxin transport. Planta 1974; 118:101-21.

[4] Raven JA. Transport of indoleacetic acid in plant cell in relation to $\mathrm{PH}$ and electrical potential gradients, and its significance for polar IAA transport. New Phytol 1975; 74:163-72.

[5] Bennett MJ, Marchant A, Green HG, May ST, Ward SP, Millner PA, et al. Arabidopsis AUX1 gene: a permeaselike regulator of root gravitropism. Nature 1996; 273: 948-50.

[6] Gälweiler L, Guan C, Müller A, Wisman E, Mendgen K, Yephremov A, Palme K. Regulation of polar auxin transport by AtPIN1 in Arabidopsis vascular tissue. Science 1998 ; 282:2226-30.

[7] Chen R, Hilson P, Sedbrook J, Rosen E, Casper T, Masson $\mathrm{PH}$. The Arabidopsis thaliana AGRAVITROPIC1 gene encodes a component of the polar-auxin-transport efflux carrier. Proc Natl Acad Sci USA 1998 ; 95:15112-7.

[8] Luschnig C, Gaxiola R, Grisafi P, Fink GR. EIR1, a root specific protein involved in auxin transport, is required for gravitropism in Arabidopsis thaliana. Genes Dev 1998; 12:2175-87.

[9] Möller A, Guan C, Gälweiler L, Tanzler P, Huijser P,
Marchant A, et al. AtPIN2 defines a locus of Arabidopsis for root gravitropism control. EMBO J 1998 ; 17:690311.

[10] Okada K, Ueda H, Komaki MK, Bell CJ, Shimura Y. Requirement of the auxin polar transport in the early stage of Arabidopsis floral bud formation. Plant Cell 1991; 3:677-84.

[11] Liu CM, Xu ZH, Chua NH. Auxin polar transport is essential for the establishment of bilateral symmetry during early plant embryogenesis. Plant Cell 1993; 5:62130 .

[12] Reinhardt D, Mandel T, Kuhlemeier C. Auxin regulates the initiation and radial position of plant lateral organs. Plant Cell 2000; 12:507-18.

[13] Vernoux T, Kronenberger J, Grandjean O. PINFORMED1 regulates cell fate at the periphery of the shoot apical meristem. Development 2000; 127:515765.

[14] Ni WM, Chen XY, Xu ZH, Xue HW. Isolation and functional analysis of a Brassica juncea gene encoding a component of auxin efflus carrier. Cell Research 2002; 12 (3):235-45.

[15] Alfandari D, Darribere T. A simple PCR method for screening cDNA libraries. PCR Methods and Applications $1994 ;$ 4:46-9.

[16] Logemann J, Schell J, Willmitzer L. Improved method for the isolation of RNA from plant tissues. Anal Biochem 1987; 163:21-6.

[17] Xue HW, Pical C, Brearley C, Elge S, Müller -Röber B. A plant 126-kDa phosphatidylinositol 4-kinase with a novel repeat structure: cloning and functional expression in baculovirus-infected insect cells. J Bio Chem 1999; 274: 5738-45.

[18] Jefferson RA, Kavanagh TA, Bevan MW. GUS fusions: bglucuronidase as a sensitive and versatile gene fusion marker in higher plants. EMBO J 1987; 6:3901-7.

[19] Jones AM. Auxin transport: down and out and up again. Science 1998; 282:2201-2.

[20] Bisseling T, Weigel D. Plant development: from cell fate to organ formation (meeting report). Plant Cell 2001; 13:222.

[21] Morelli G, Ruberti I. Shade avoidance responses. Driving auxin along lateral routes. Plant Physiol 2000; 122:6216.

[22] Sieburth LE. Auxin is required for leaf vein pattern in Arabidopsis. Plant Physio 1999; 121:1179-90.

[23] Mattsson J, Sung ZR, Berleth T. Response of plant vascular systems to auxin transport inhibition. Development 1999; 126:2979-91.

[24] Nemhauser JL, Feldman LJ, Zambryski PC. Auxin and ETTIN in Arabidopsis gynoecium morphogenesis. Development 2000; 127:3877. 